\title{
Chromosomal copy number variation reveals differential levels of genomic plasticity in distinct Trypanosoma cruzi strains
}

João Luís Reis-Cunha', Gabriela F. Rodrigues-Luiz', Hugo O. Valdivia', Rodrigo P. Baptista', Tiago A. O. Mendes', Guilherme Loss de Morais ${ }^{2}$, Rafael Guedes ${ }^{2}$, Andrea M. Macedo ${ }^{3}$, Caryn Bern ${ }^{4}$, Robert H. Gilman ${ }^{5,6}$, Carlos Talavera Lopez ${ }^{7}$, Björn Andersson ${ }^{7}$, Ana Tereza Vasconcelos ${ }^{2}$ and Daniella C. Bartholomeu ${ }^{1 *}$

\begin{abstract}
Background: Trypanosoma cruzi, the etiologic agent of Chagas disease, is currently divided into six discrete typing units (DTUs), named TCl-TCVI. CL Brener, the reference strain of the T. cruzi genome project, is a hybrid with a genome assembled into 41 putative chromosomes. Gene copy number variation (CNV) is well documented as an important mechanism to enhance gene expression and variability in T. cruzi. Chromosomal CNV (CCNV) is another level of gene CNV in which whole blocks of genes are expanded simultaneously. Although the T. cruzi karyotype is not well defined, several studies have demonstrated a significant variation in the size and content of chromosomes between different $T$. cruzi strains. Despite these studies, the extent of diversity in CCNV among T. cruzi strains based on a read depth coverage analysis has not been determined.
\end{abstract}

Results: We identify the CCNV in T. cruzi strains from the Tcl, Tcll and TcIll DTUs, by analyzing the depth coverage of short reads from these strains using the $41 \mathrm{CL}$ Brener chromosomes as reference. This study led to the identification of a broader extent of CCNV in T. cruzi than was previously speculated. The TCI DTU strains have very few aneuploidies, while the strains from Tcll and TcIll DTUs present a high degree of chromosomal expansions. Chromosome 31, which is the only chromosome that is supernumerary in all six T. cruzi samples evaluated in this study, is enriched with genes related to glycosylation pathways, highlighting the importance of glycosylation to parasite survival.

Conclusions: Increased gene copy number due to chromosome amplification may contribute to alterations in gene expression, which represents a strategy that may be crucial for parasites that mainly depend on post-transcriptional mechanisms to control gene expression.

Keywords: Chromosome copy number variation, Trypanosoma cruzi, Genomic plasticity

\section{Background}

American trypanosomiasis is a neglected tropical disease, caused by the protozoan Trypanosoma cruzi, a highly polymorphic parasite that belongs to the order Kinetoplastida and family Trypanosomatidae. The distribution of this disease ranges from southern Argentina to the southern United States of America, where it affects

\footnotetext{
* Correspondence: daniella@icb.ufmg.br

'Laboratório de Imunologia e Genômica de Parasitos, Departamento deParasitologia, Universidade Federal de Minas Gerais, Belo Horizonte, Brazil Full list of author information is available at the end of the article
}

eight million people and accounts for 662,000 disabilityadjusted life years [1-4].

The $T$. cruzi taxa is currently subdivided into six discrete typing units (DTUs), named TcI - TcVI, due to its high genotypic and phenotypic heterogeneity. The major T. cruzi DTUs involved in the domestic cycle of Chagas disease are TcI, TcII, TcV and TcVI [5-11]. The distinct DTUs are differently distributed in the Americas, with TcI prevalent in Central America and in the northern region of South America, while TcII, TcV and TcVI are more common in the Southern cone of South America [10]. 
T. cruzi replication is usually clonal $[12,13]$, but there is evidence of natural hybridization and genetic exchange between the strains [14-19]. The hybrid nature of T. cruzi DTU TcVI was confirmed during the wholegenome sequencing of the TcVI CL Brener clone [20]. Post-assembly comparisons of CL Brener contigs with reads from the $T$. cruzi Esmeraldo TcII strain allowed the differentiation of the two CL Brener haplotypes, named Esmeraldo-like, derived from a TcII ancestor, and non-Esmeraldo-like, derived from a TcIII ancestor [20].

Apparently, TcI has the smallest genome of all the T. cruzi DTUs $[6,21,22]$, and it seems to have less intragenomic heterogeneity than TcII and TcVI [23]. However, sequences from different TcI strains may present more sequence variability between each other than the variability within the TcII and TcVI strains [8, 23].

Copy number variation (CNV) - the gain or loss of genomic material-may have a phenotypic impact by altering the fitness of an organism. CNV creates paralog genes that may evolve differently than the progenitor gene or that may alter the expression level of a gene or genomic region [24, 25]. In CL Brener, at least $50 \%$ of the genome consists of repetitive sequences, represented primarily by large multigene families that encode surface proteins, retrotransposons, and telomeric and satellite repeats $[20,26]$. The CL Brener genome contains approximately 1000 paralogous clusters with more than two genes, encompassing over 8000 genes. Several of these clusters are represented by surface protein-encoding genes that account for $18 \%$ of the total of protein-encoding genes of CL Brener [20]. An increased gene copy number due to chromosomal amplification may contribute to alterations in gene expression, providing a strategy for organisms, such as $T$. cruzi, that depend mainly on post-transcriptional mechanisms to control gene expression [27, 28].

The $T$. cruzi karyotype has not been completely elucidated owing to the inability to perform cytogenetic analysis because there is no apparent chromosome condensation during the parasite cell cycle [8, 22, 29]. Using pulse-field gel electrophoresis (PFGE), various studies have shown a significant variation in chromosome size and content between different T. cruzi strains and even between clones of the same strain [5, 29-32]. To better characterize the $T$. cruzi karyotype, the genome sequence of the CL Brener strain was recently assembled into 41 putative chromosomes based on scaffold information, BAC-end sequences, and synteny maps with Trypanosoma brucei and Leishmania major [8, 33].

Read depth coverage (RDC) analysis allows the identification of extensive variations in the copy number of chromosomes among different species of Leishmania [34]. However, the chromosomal copy number variation (CCNV) among T. cruzi strains as determined by read depth coverage analysis has not yet been reported. In the present work, we sought to identify the CCNV in T. cruzi strains belonging to different DTUs, based on read depth coverage of the $41 \mathrm{CL}$ Brener chromosomes. Identifying the CCNV will lead to explanations of some of the genome structural peculiarities of these DTUs. This analysis also led to the identification of a broader extent of CCNV in $T$. cruzi than previously speculated, especially in strains from the TcII and TcIII DTUs.

\section{Results}

\section{Competitive mapping and SNP content}

To select the CL Brener haplotype most suitable as a reference in the mapping of the reads from the distinct T. cruzi strains, their quality-filtered reads were mapped simultaneously to the 41 chromosomal sequences from the CL Brener Esmeraldo-like and non-Esmeraldo-like haplotypes (Fig. 1a). As expected, the reads from the TcII strains (Esmeraldo and Y) mapped preferentially with chromosomes from the Esmeraldo-like haplotype, and the reads from the TcIII strain (231) mapped preferentially with chromosomes from the non-Esmeraldo-like haplotype. The reads from the strains from TcI DTU (Arequipa, Colombiana and Sylvio) mapped slightly better to the non-Esmeraldo-like than to the Esmeraldo-like CL Brener haplotype (Fig. 1b).

To further confirm the selection of the haplotype to be used as a reference for read mapping, the filtered reads from each strain were mapped separately to both CL Brener haplotypes, and then the number of SNPs/1000 bp in the CL Brener single-copy genes for each combination of strain-haplotype was estimated (Fig. 1c). The numbers of SNPs/1000 bp between each T. cruzi strain and the CL Brener Esmeraldo-like and non-Esmeraldo-like haplotypes were, respectively, 17.50 and 3.10 for Arequipa; 32.74 and 14.37 for Colombiana; 52.41 and 25.36 for Sylvio; 5.66 and 18.07 for Esmeraldo; 4.39 and 16.08 for Y; and 17.89 and 5.72 for 231.

Based on these results, the CL Brener non-Esmeraldolike haplotype was selected as a reference for the mapping of Arequipa, Colombiana, Sylvio and 231 reads, and the CL Brener Esmeraldo-like haplotype was selected as a reference for the mapping of Esmeraldo and $Y$ strain reads.

\section{Methodology to estimate chromosome copy number in T. cruzi strains}

Approximately $50 \%$ of the $T$. cruzi genome corresponds to repetitive sequences, including multigene families that account for much of the differences in the gene content of the assembled genomes of CL Brener (TcVI) and Sylvio (TcI) [20, 21]. The chromosomal sequence representation of the CL Brener non-Esmeraldo-like and Esmeraldo-like haplotypes [33] also contains large internal gap regions 


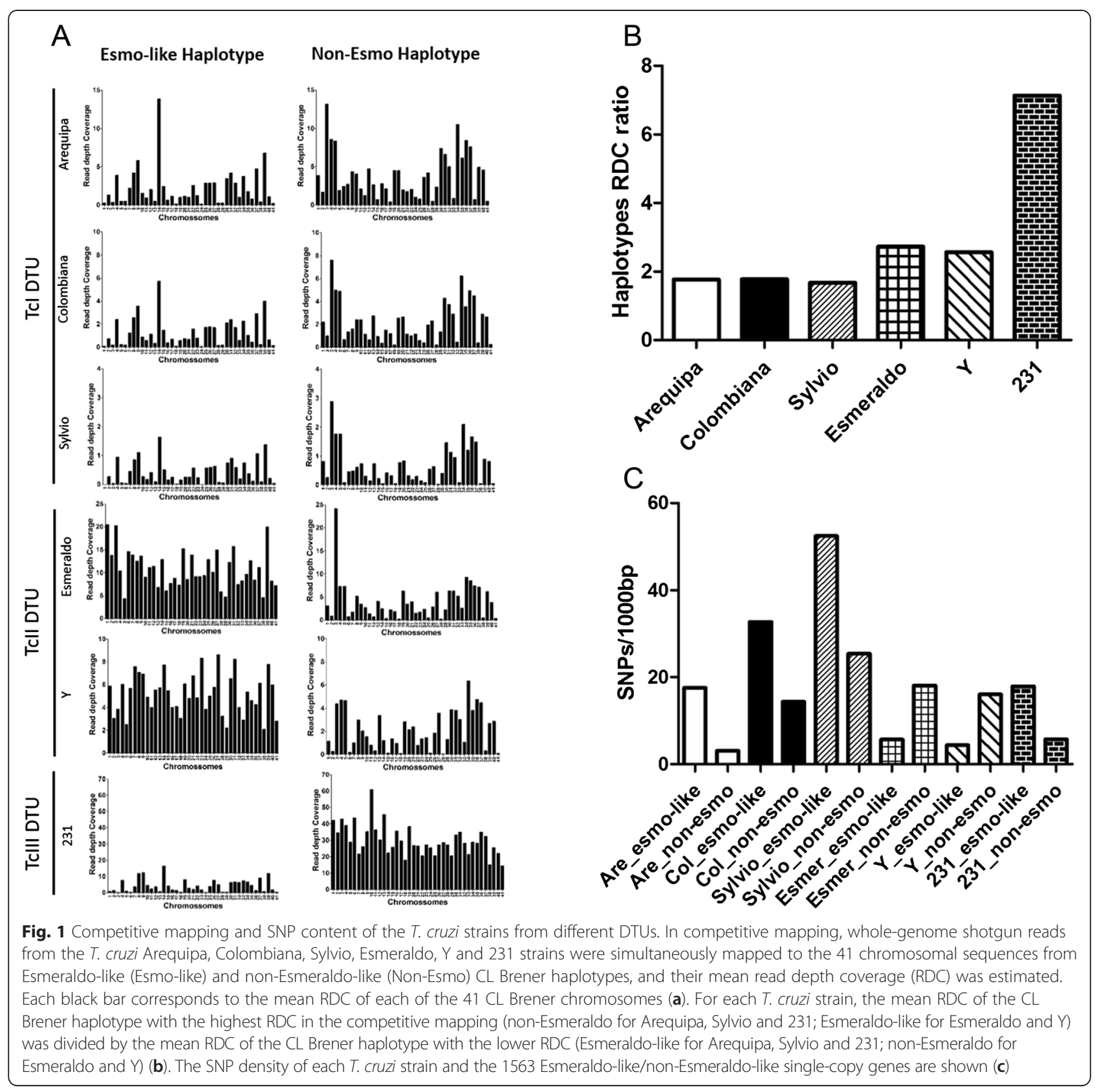

that may reduce the accuracy of the predicted ploidy based on RDC. To reveal the best methodology to determine the T. cruzi chromosome ploidy based on RDC, two approaches were evaluated. In the Whole Chromosome Ploidy Estimations (WCPE) approach, the chromosomal ploidy prediction for each chromosome was estimated based on the ratio between the mean RDC of each chromosome position and the genome coverage (Fig. 2a). This approach accounts for the coverage of all positions in a given chromosome to estimate its copy number, including repetitive and gap regions. In the single-copy genes ploidy estimations (SCoPE) approach, estimations of the chromosomal ploidy for each chromosome were based on the ratio between the mean coverage of all single-copy genes in a given chromosome and the genome coverage (Fig. 2b). This approach infers the copy number for each chromosome based only on the RDC of the $15631: 1$ orthologs between CL Brener Esmeraldo-like and nonEsmeraldo-like haplotypes, which were assumed to be single-copy genes in the haploid CL Brener genome content (Additional file 1: Table S1). As shown in Fig. 2, chromosomes that are rich in multigene families, repetitive sequences and gaps, such as chromosomes 18, 28, 38 and 41 (Fig. 2c), tend to have a lower predicted ploidy as determined using WCPE methodology when compared to the SCoPE approach using the Y strain reads. Similar results 


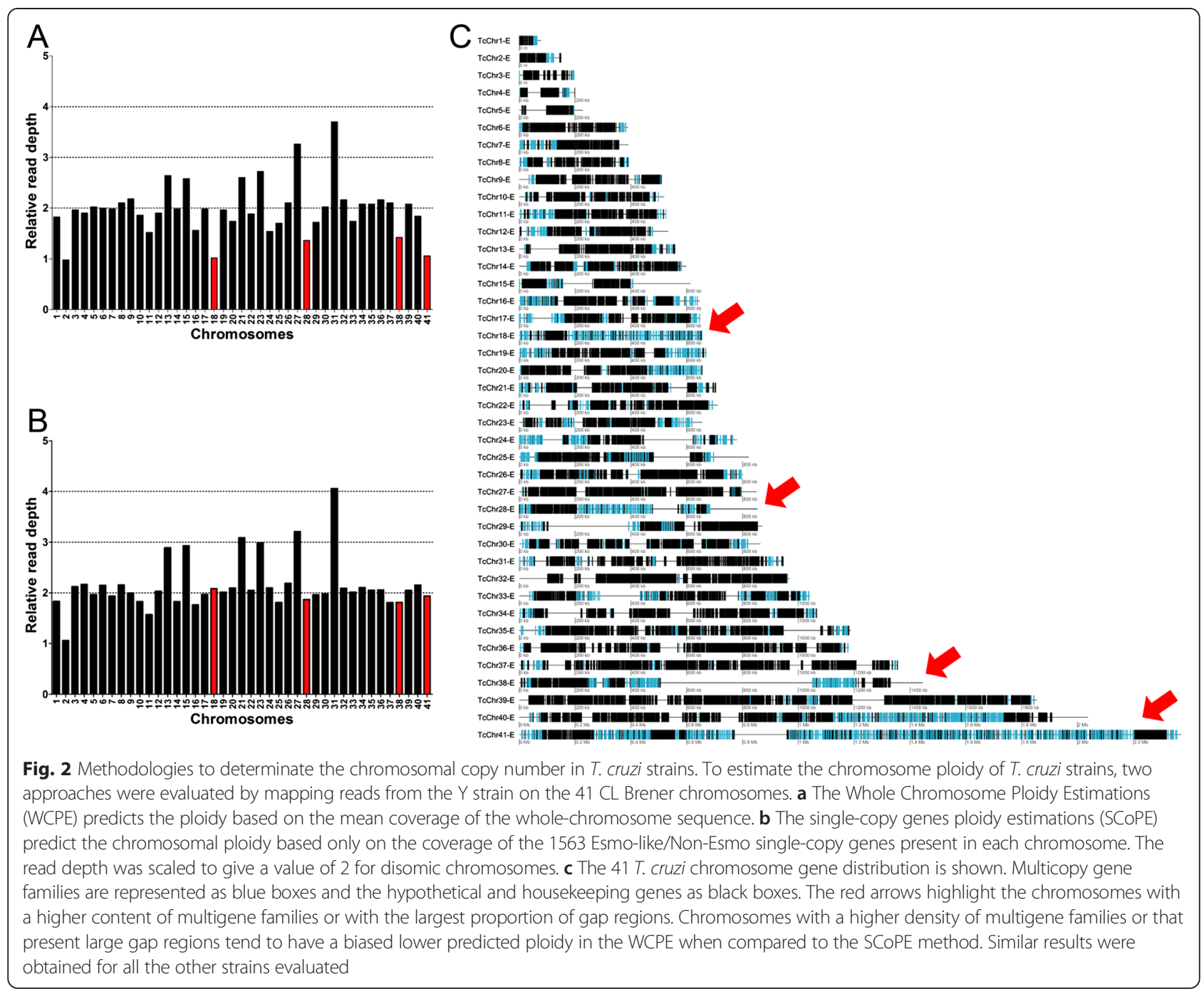

were obtained by mapping reads from the other $T$. cruzi strains on the $41 \mathrm{CL}$ Brener chromosomes (data not shown). As the SCoPE approach is less prone to bias toward chromosomal repetitive content, this methodology was chosen to estimate the chromosomal ploidy for each of the strains used in this study.

\section{Chromosome copy number variation in $T$. cruzi strains}

The SCoPE approach was used to estimate the chromosome ploidy of the T. cruzi Arequipa, Colombiana, Sylvio, Esmeraldo, Y and 231 strains. Initially, based on the mean RDC of 1563 CL Brener single-copy genes, the genome coverage was estimated for each strain: $47 \times$ for Arequipa, 28× for Colombiana, $9 \times$ for Sylvio, $52 \times$ for Esmeraldo, $34 \times$ for $\mathrm{Y}$ and $76 \times$ for 231 . The normalized read depth coverage and the percentage of length coverage of each single-copy gene in each chromosome are provided in Additional file 2: Table S2. To determine the overall chromosome ploidy of each $T$. cruzi strain, the allele frequencies were estimated for each predicted heterozygous site. To this end, the proportion of each allele in the heterozygous sites divided by the total read depth for the site was determined and rounded to the first decimal place. Based on this estimation, a diploid chromosome usually has a tendency of 0.4-0.5 and a triploid of 0.3 and 0.6. A tetraploid chromosome has a more complex pattern, which can be $0.4-0.5,0.2$ and 0.8 or a combination of both. As the majority of the heterozygous SNPs show a proportion of 0.4-0.5, the overall ploidy of all the strains was assumed to be diploid (Fig. 3).

The chromosome CNV analysis revealed large differences between the $T$. cruzi strains from different DTUs and some differences between members of the same DTU. Apparently, strains from the TcI DTU have a more stable karyotype when compared to strains from the TcII and TcIII DTUs (Fig. 3). Strains from the TcI DTU usually only have an aneuploidy in chromosome 31 , with 


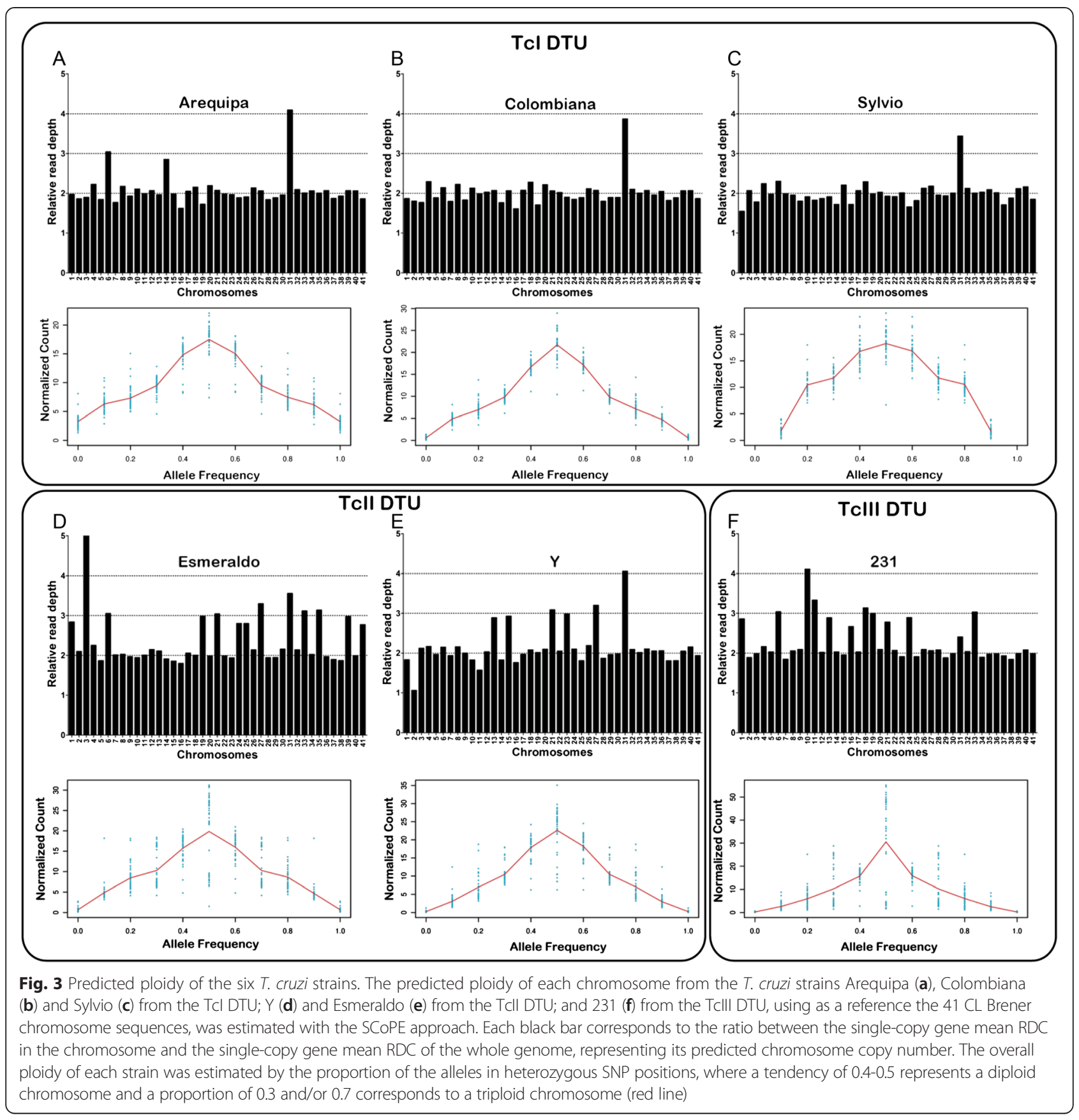

the exception of the Arequipa strain, which also has a trisomy in chromosomes 6 and 14. The strains from the TcII DTU have a more plastic karyotype with several predicted supernumerary chromosomes and a monosomy case. The Esmeraldo strain has chromosome 3 as pentasomic; chromosomes 27, 31, 33 and 35 range from trisomic to tetrasomic; chromosomes 6, 19, 21 and 39 as trisomic; and chromosomes 1, 24, 25 and 41 range from disomic to trisomic. The Y strain, also from TcII DTU, has tetrasomy of chromosome 31; chromosomes 21 and 27 range from trisomic to tetrasomic; trisomy of chromosomes 13, 15, and 23; and monosomy of chromosome 2. The representative of TcIII DTU, 231, has tetrasomy of chromosome 10; chromosomes 11 and 18 range from trisomic to tetrasomic; trisomy of chromosomes 6 and 19; and chromosomes 1, 13, 16, 21, 24 and 31 have a ploidy ranging from disomic to trisomic (Fig. 3).

To further confirm the chromosomal ploidy, the distribution of base frequencies between the heterozygous SNP positions among all the CDSs in the 41 chromosomes of the six T. cruzi strains were estimated (Additional file 3: Figure S1). This analysis is in agreement with the CCNV 
results predicted by the SCoPE methodology. The only exceptions were chromosomes 20 and 23 from Sylvio and chromosome 7 from Esmeraldo, which were predicted as tetraploid by the heterologous SNP proportion and as diploid in the SCoPE analysis, and chromosomes 6 and 14 from Arequipa that were tetraploid by the heterologous SNP proportion and triploid in the SCoPE analysis.

To evaluate if these predicted aneuploidies were produced by the gain or loss of a whole chromosome, or if they result from segmental duplication or loss of partial fragments from these chromosomes, the normalized read depth coverage of each position along each chromosome of the six T. cruzi strains was estimated (Additional file 4: Figure S2). Figure 4 represents the read depth coverage along each position of the predicted disomic, trisomic and tetrasomic chromosomes of the Y strain, as well as the base frequency distribution between the heterozygous
SNP positions. As expected, with the exception of the regions that are rich in multigene families and gaps, the predicted ploidy along the entire chromosome is in agreement with the predicted ploidy based on the SCoPE and SNP analyses. This finding suggests that these aneuploidies are probably a result of a whole chromosomal duplication/loss.

To determine whether the ploidy profile of the six T. cruzi strains is in agreement with their phylogeny, a hierarchical clustering analysis was performed, using as input the predicted ploidy of each chromosome of each strain based on the SCoPE approach. The clustering analysis was based on their pairwise Euclidean distances using the Complete Linkage method (Fig. 5). In this analysis, all strains belonging to the TcI DTU clustered together, with Colombiana and Sylvio strains being closer to each other than to the Arequipa strain. This is likely

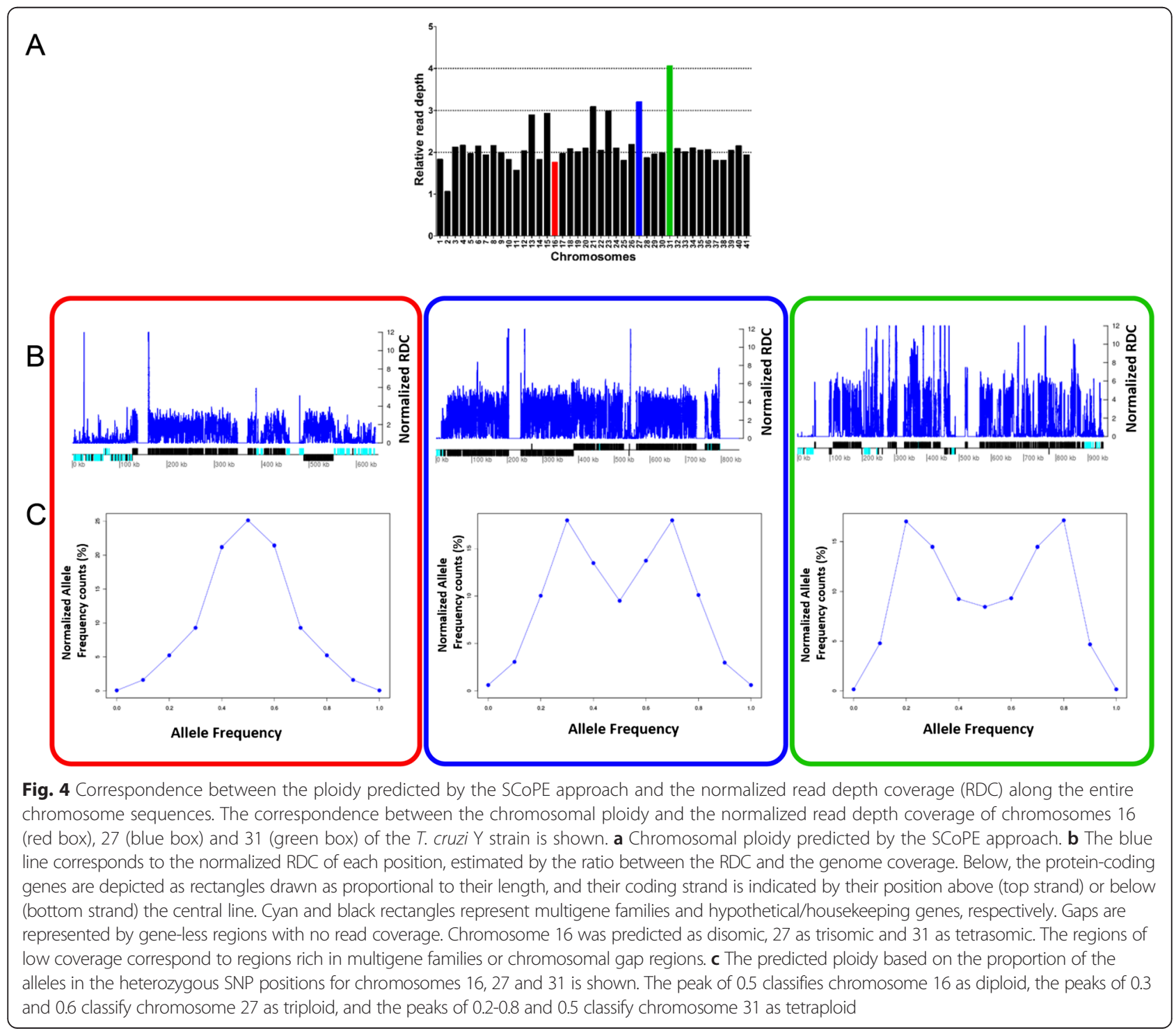




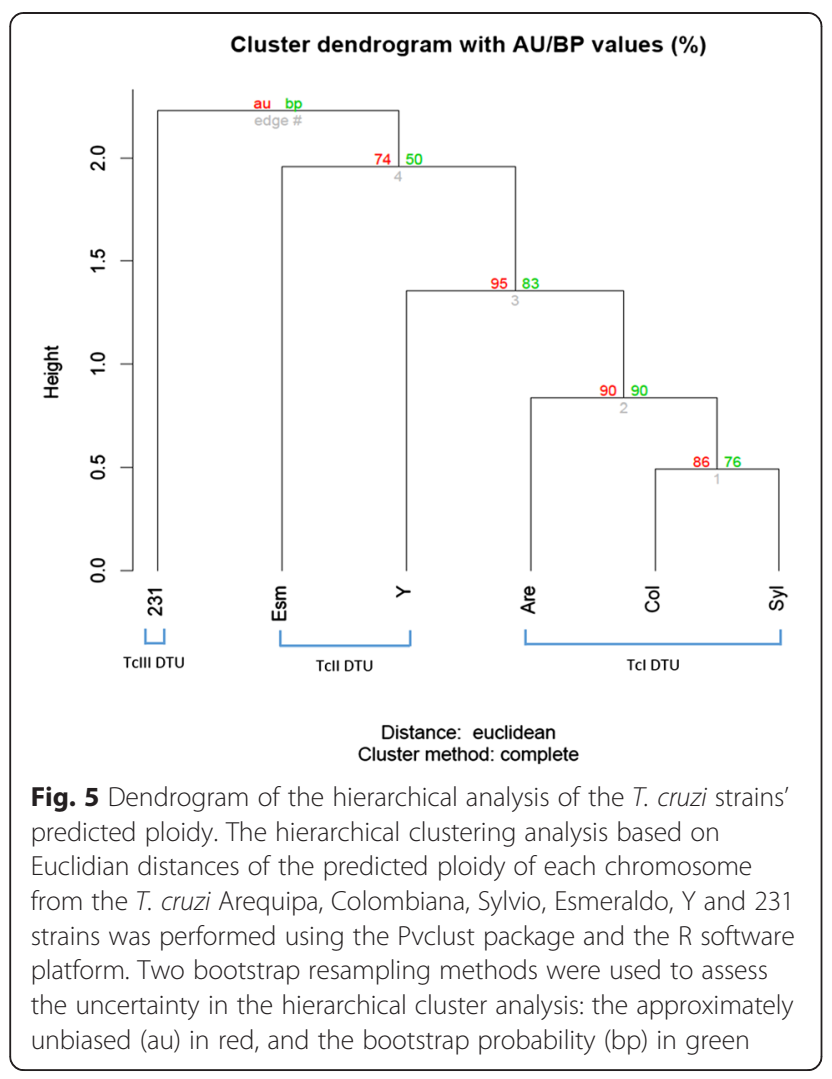

due to the presence of exclusive aneuploidies in chromosomes 6 and 14 from Arequipa. Both TcII DTU strains showed a different pattern of chromosomal aneuploidies from each other and showed a smaller group consistency than the strains from the TcI DTU. The 231 TcIII DTU strain shows the most different pattern among the T. cruzi strains evaluated.

\section{Y Chromosome 11}

Chromosome 11 from the $\mathrm{Y}$ strain has some interesting features. As shown in Fig. 3, this chromosome displayed a smaller ploidy than disomic but a greater ploidy than monosomic. This led us to investigate whether this pattern occurs due to the loss of a chromosomal region instead of the loss of a chromosome copy. As showed in Fig. 6, there is a drastic change in the RDC starting at the 248-kb position in this chromosome that corresponds to a strand switch region. The first $248 \mathrm{~kb}$ of this chromosome has a smaller predicted ploidy when compared to the rest of the chromosome sequence (Fig. 6a). To further confirm this chromosomal region loss, the mean RDC of the single-copy genes located upstream and downstream of the $248-\mathrm{kb}$ coordinate were estimated. The single-copy genes that were upstream of the $248-\mathrm{kb}$ position had a non-normalized mean RDC of 20 , while the single-copy genes downstream had an approximate mean RDC of 40 (Fig. 6b). This reduction of the
RDC in the $5^{\prime}$ region when compared to the rest of the chromosome and the estimated genome coverage of $34 \times$ suggest a segmental loss of the $248 \mathrm{~kb}$ at the $5^{\prime}$ region in one copy of this chromosome in the Y strain. Alternatively, this pattern may be due to differences in the structure of chromosome 11 among the different cells of the parasite population because $\mathrm{Y}$ is not a cloned line.

\section{Chromosome 31 gene ontology}

From all the $41 \mathrm{CL}$ Brener chromosomes, chromosome 31 was the only one that was supernumerary in all the strains analyzed, in both SCoPE (Fig. 7a) and heterozygous SNP analyses (Fig. 7b). To identify gene functions that were overrepresented in this chromosome when compared to the whole genome, a gene ontology analysis of both Esmeraldo-like and non-Esmeraldo-like CL Brener chromosome 31 was performed (Fig. 7c, Additional file 5: Table S3). This analysis shows that this chromosome is enriched in genes involved in glycosylation and glycoprotein biosynthetic processes in both CL Brener haplotypes.

\section{Discussion}

The availability of the sequence representation of the 41 putative chromosomes from both Esmeraldo-like (TcII) and non-Esmeraldo (TcIII) CL Brener haplotypes [33] allowed a comparative analysis of $T$. cruzi strain ploidy using the unassembled next generation sequencing reads. However, the repetitive nature of the $T$. cruzi genome along with the presence of gap regions in the sequence representation of CL Brener chromosomes hampers the effectiveness of CCNV comparisons based on the read depth coverage (RDC) of whole-chromosome sequences. To overcome these limitations, we propose the SCoPE approach that is based on the RDC of single-copy genes that are conserved between Esmeraldo-like and non-Esmeraldo-like haplotypes as well as in the six T. cruzi strains evaluated in this study (Additional file 2: Table S2). These single-copy genes were used as chromosomal markers for unique genomic sequences to normalize the CCNV estimations, revealing large differences in chromosomal copy number between T. cruzi strains (Fig. 3).

It is well known that T. cruzi strains present a distinct profile of chromosomal bands, which present different sizes and numbers in Pulse Field Gel Electrophoresis, suggesting a variable karyotype among the DTUs. These differences were mainly attributed to a differential repetitive content in the genomes of distinct $T$. cruzi strains, or to chromosomal fusion/break events during the parasite evolution [5, 22, 30, 31, 35]. In fact, approximately $50 \%$ of the CL Brener genome corresponds to repetitive sequences, many of them clustered into regions containing multigene families encoding surface proteins and transposable elements [20,33]. These clusters are extremely 


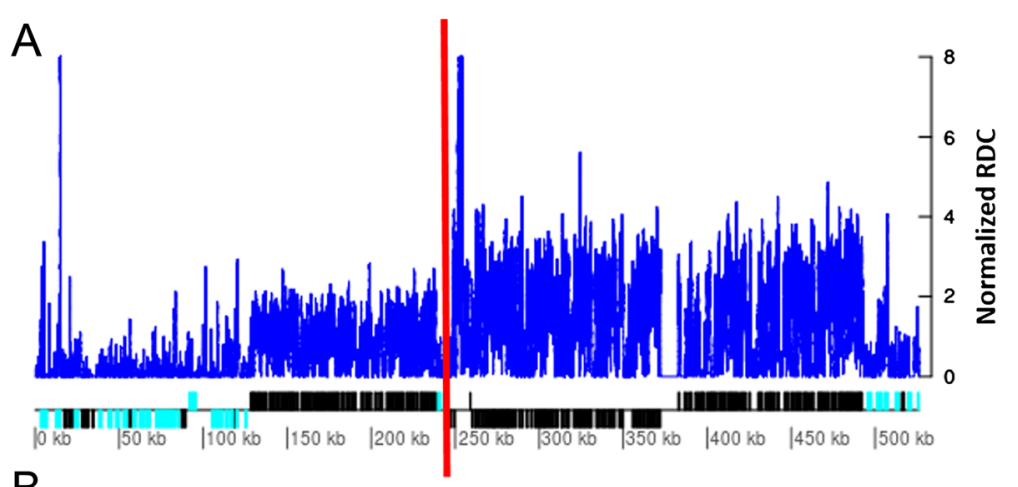

B

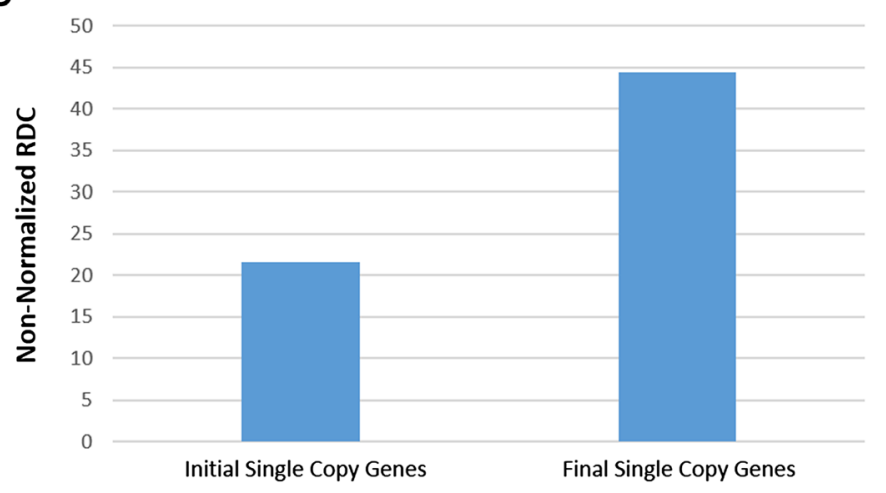

Fig. 6 Predicted ploidy of chromosome 11 of the T. cruzi Y strain. The blue line corresponds to the normalized RDC of each position of chromosome 11 , estimated by the ratio between the RDC and the genome coverage. Below, the protein-coding genes are depicted as rectangles drawn in proportion to their length, and their coding strand is indicated by their position above (top strand) or below (bottom strand) the central line. Black and cyan rectangles represent the housekeeping/hypothetical and multicopy gene families, respectively. Gaps are represented by gene-less regions with no read coverage. The red line corresponds to the 248-kb position in this chromosome, which separates the regions of low RDC and high RDC (a). The non-normalized mean RDC estimated based on the SCOPE approach of the genes that were upstream and downstream of the 248-kb position was estimated (b)

variable in their gene content and length and are regions of synteny loss when comparing the two CL Brener haplotypes (El-Sayed 2005a) and the CL Brener and Sylvio genomes [21]. Despite these variations, single-copy genes are, in general, highly conserved and syntenic among the T. cruzi strains $[8,21,22,36]$ (Baptista in preparation) and therefore represent an adequate source for sequence normalization in comparative CCNV analyses.

The occurrence of whole chromosome aneuploidy in T. cruzi was previously suggested based on whole genome oligonucleotide tiling arrays and competitive genomic hybridization between 16 T. cruzi strains and CL Brener clone as reference [8]. However, the absolute number of individual chromosomes in each strain was not estimated [8]. Also, the comparative genomic hybridization analysis does not allow the detection of chromosomal expansion/ deletions that are present simultaneously in CL Brener and in the tested strain [8]. Our approach, however, used the haploid content of CL Brener chromosomes as reference for read mapping, allowing the detection of chromosomal copy number variations that may be also present in CL Brener.

\section{Competitive mapping}

To identify the CL Brener haplotype suitable to be used as a reference, each $T$. cruzi strain read collection from the TcI (Arequipa, Colombiana and Sylvio), TcII (Esmeraldo and Y) and TcIII (231) strains was simultaneously mapped to both Esmeraldo-like and non-Esmeraldo-like CL Brener haplotypes, using a competitive approach (Fig. 1a). As the mapping quality cutoff was very stringent, the conserved regions that mapped simultaneously to both CL Brener haplotypes were excluded, and only reads that mapped preferentially to one haplotype were considered. As expected, reads from the TcII strains, namely Esmeraldo and Y, mapped better to the Esmeraldo-like haplotype (CL Brener haplotype derived from a TcII ancestor genome), and reads from TcIII mapped better to the non-Esmeraldo haplotype (derived from the TcIII ancestor) (Fig. 1b). All strains belonging to the TcI DTU-Arequipa, Colombiana and Sylvio-mapped slightly better to the non-Esmeraldo haplotype, suggesting a closer proximity to TcI compared to TcIII as previously described $[11,14,15,21]$. Interestingly, all TcI strains had a higher RDC for the Esmeraldolike chromosome 14 than for the same chromosome from 


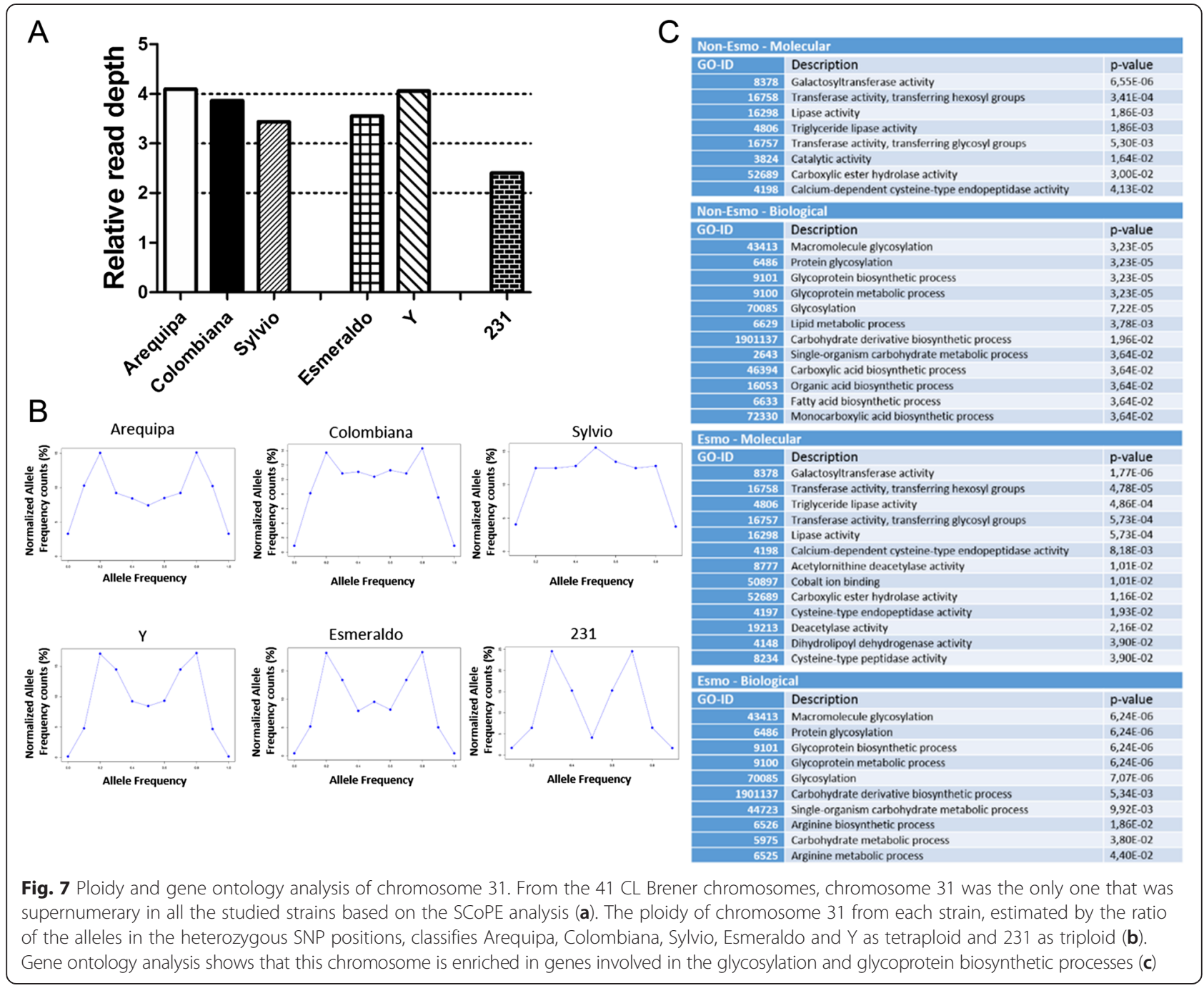

the non-Esmeraldo haplotype. Another example is the Esmeraldo chromosome 3, which had a slightly better RDC for the non-Esmeraldo than for the Esmeraldo-like. A careful inspection of the RDC along the entire sequences of these chromosomes revealed that this unexpected profile was due to an extremely high RDC of a specific region in both chromosomes. In the case of chromosome 14, the genomic region encompassing the gene Tc00.10470535007099.80 (ABC transporter putative) in the Esmeraldo-like haplotype displays a very high RDC, and this region is absent in the non-Esmeraldo-like haplotype. Therefore, it is likely that this is a repetitive sequence where reads from both CL Brener haplotypes were collapsed in the assembled Esmeraldo-like haplotype. Likewise, in the case of chromosome 3, approximately 1500 nucleotides of the $5^{\prime}$ end of the gene Tc00.1047053508533.10 (hypothetical protein conserved) from the non-Esmeraldolike haplotype are missing in the corresponding allele (Tc00.1047053404001.20) of the Esmeraldo-like haplotype.
These genes are not included in the 1563 copy genes used by SCoPE to estimate the CCNV, further validating this methodology.

\section{Chromosomal copy number variation and strain ploidy}

All six T. cruzi strains evaluated in this study had an overall genome ploidy predicted as diploid, based on both the SCoPE approach and the allele proportion in heterozygous positions, when the whole genome was evaluated (Fig. 3). This result is in agreement with previous estimations for T. cruzi $[8,9,37]$ and Leishmania species that classified these parasites as diploid, with the exception of L. braziliensis (M2904) that had an overall trisomic chromosomal pattern [34].

The CCNV among T. cruzi strains was initially estimated by the SCoPE approach (Fig. 3). The strains from the TcI DTU (Colombiana, Sylvio and Arequipa) had a low number of whole chromosome expansions $(1,1$ and 3 , respectively), while the TcII strains (Esmeraldo and Y) 
and the TcIII strain (231) had a larger number of these expansions (13, 6 and 12, respectively). These predictions were further confirmed by the heterologous SNP analysis, which predicted the same aneuploidies, with the exception of chromosomes 20 and 23 from Sylvio, 7 from Esmeraldo and 6 and 14 from Arequipa that were predicted as tetrasomic. In these chromosomes, the heterozygous SNP analysis predicted an even broader chromosomal expansion than those estimated by the SCoPE approach. To evaluate if the CCNV predicted using the single-copy genes was not impacted by segmental duplications or loss of partial fragments from these chromosomes, we estimated the RDC of all the positions from all 41 chromosomes of the six T. cruzi strains (Fig. 4, Additional file 4: Figure S2). Excluding chromosomal gaps and regions containing clusters of multigene families, the RDC of the whole chromosome is in agreement with the SCoPE CCNV prediction, validating this approach (Fig. 4).

Gene copy number variation is well documented as an important mechanism to enhance gene expression and variability not only in $T$. cruzi, but also in T. brucei and Leishmania $[8,20,28,36,38-40]$. The CCNV arises as a new level of CNV, expanding whole blocks of genes simultaneously [34]. The occurrence of CCNV was already associated to increased fitness in stress conditions and to drug resistance in Saccharomyces cerevisiae and Candida albicans [41-43]. The karyotype plasticity and CNV found in $T$. cruzi could also represent a framework for the natural selection of favorable phenotypes, such as higher expression of virulence-factors and increased diversity, resulting in an enhanced adaptability of the parasite $[8,44]$.

The mechanisms involved in the generation of $\mathrm{CCNV}$ in T. cruzi are still unknown. Even though it has been previously proposed that meiosis events occur in Trypanosoma brucei [45] and Leishmania [46], this process has not been demonstrated in T. cruzi. However, a nonmeiotic hybridization model has been proposed to explain the generation of hybrid T. cruzi DTUs [47]. According to this model, during the mammalian stage of the parasite, the nucleus of two diploid cells fuse, resulting in a polyploidy progeny that can undergo recombination between alleles. This new polyploid cell may lose some of its supernumerary chromosomal copies, eventually returning to the diploid state [48]. This could be one of the mechanisms related to the generation of CCNV in T. cruzi, as some of these extra chromosomal copies may be maintained by the parasite.

For all the T. cruzi DTUs evaluated, TcI had the fewest number of chromosomal expansions, exhibiting the most stable karyotype (Fig. 3). The reduced number of chromosomal duplications could be a contributing factor for the low levels of heterozygosity found in TcI [21, 49].
It has been demonstrated that the mismatch repair machinery, which repairs base miss-incorporation and erroneous insertions and deletions during DNA recombination and replication, is more efficient in TcI than in the other DTUs and is a known factor in the reduction of heterozygosity in TcI $[50,51]$. TcI strains also have the smallest genome size compared to the other T. cruzi DTUs [6], which were associated with the reduction of multigene family clusters within this DTU [21]. Based on our results, we propose that this reduction in genome size is also associated with the lower number of chromosomal duplications in TcI when compared to other DTUs. Similarly, data from Rogers 2011 showed a correlation between CCNV based on RDC analysis and DNA content estimated using flow cytometry in different Leishmania species [34]. Variation in DNA content among T. brucei species and isolates was also observed [52] and could be related with some level of CCNV in this parasite, which so far has not been formally demonstrated. Although TcI strains have less intragenomic heterogeneity, sequences belonging to different strains are more distant from each other within TcI than within TcII and TcVI $[8,23]$. This increased variation suggests a reduced number of genetic exchange events among strains from the TCI DTU, which could result in a lower rate of chromosomal ploidy variations.

For the TcII DTU, Esmeraldo had 13 chromosomal expansions while $Y$ had 6 , suggesting that there are extensive differences in chromosomal duplications within TcII DTU (Fig. 3). Recently, substantial recombination and genetic exchange among strains from the TcII DTU that coexist in the same geographical area was proposed based on microsatellite genotype data [19]. The broader chromosomal expansion in Esmeraldo may be explained by the fact that haplotypes that constitute this strain are more distant from each other than the ones that constitute $Y$ [15], which suggests that Esmeraldo suffered more recombination events than $\mathrm{Y}$, making it more susceptible to acquiring aneuploidies [15, 48]. Leishmania isolates also display a broad range of CCNV. As TcI DTU strains evaluated in this study, different strains of $L$. major (Friedlin and LV39) had the same CCNV pattern. On the other hand, as observed in TcII DTUs, strains from both $L$. mexicana and $L$. donovani had extensive CCNV within the species [34]. It will be interesting to compare the efficiency of processes associated with the maintenance of genomic stability in T. cruzi DTUs and Leishmania species, and investigate the occurrence of CCNV in T. brucei, which could help to elucidate the mechanisms behind the $\mathrm{CCNV}$ in these parasites. Widespread aneuploidy found in T. cruzi and Leishmania, implies that caution should be taken when selecting markers for population genetic studies based on the hypothesis of diploidy [13]. In this case, it would be imperative selecting markers from genomic regions known to be diploid. 
Hierarchical clustering analysis based on the predicted ploidy of each T. cruzi chromosome clustered all TcI strains together with high confidence scores, further confirming the genome structural stability within DTUI (Fig. 5). Aside from the TcI DTU, strains from the TcII and TcIII DTUs had a variable CCNV pattern, suggesting a higher genome plasticity between and within these DTUs. It is interesting that the two strains from the TcII DTU (Esmeraldo and Y) had a different pattern of chromosomal ploidy, suggesting that chromosomal expansions are highly variable and may have originated several times during the evolution of the TcII DTU. The analysis of a broader number of strains would be required to correctly estimate the ratio of $\mathrm{CCNV}$ within and between the T. cruzi DTUs.

\section{Y chromosome 11}

In the first $248 \mathrm{~kb}$ of the $\mathrm{Y}$ chromosome 11, we identified 22 single-copy genes, which had half the mean RDC compared with the remaining chromosome sequence, which contains 15 single-copy genes (Fig. 6). This large change in RDC starts in a strand switch region, which is frequently associated with rearrangements in Trypanosomatid genomes $[36,53]$. This finding suggests that $Y$ chromosome 11 could have an arm loss. Another possibility is that the CL Brener chromosome 11 is divided into two distinct chromosomes in $\mathrm{Y}$, chromosomes 11a and $11 \mathrm{~b}$, at the $248-\mathrm{kb}$ position, where $11 \mathrm{a}$ is haploid and $11 \mathrm{~b}$ is diploid. Events of chromosomal break or fusion may explain the variable band pattern in PFGE among the $T$. cruzi strains [5, 22, 30-32, 35]. These events are easily detected in RDC analysis when there are large aneuploidies between fragments of the same chromosome. Alternatively, because $\mathrm{Y}$ is not a cloned population, this result may represent a mosaic structure of the parasite population, where some cells may have the entire chromosome 11 sequence while other cells may have an arm loss.

\section{Chromosome 31}

Chromosome 31 was the only one that was supernumerary in all six T. cruzi samples evaluated in this study (Fig. 7). Gene ontology analysis showed that this chromosome has an enhanced number of genes related to glycoprotein biosynthesis and glycosylation processes (Fig. 7c). CL Brener has approximately 100 genes annotated as putative glycosyltransferase genes, which are involved in the synthesis of a variety of glycoconjugates and are abundantly and differentially expressed in all T. cruzi stages [54]. From these 100 genes, 54 are UDPGlcNAc-dependent glycosyltransferases. Chromosome 31 has 9 of the 27 UDP-GlcNAc-dependent glycosyltransferase gene copies in the CL Brener Esmeraldo-like haplotype and 13 of the 27 copies in the non-Esmeraldo haplotype. This enzyme is involved in the transfer of $\mathrm{N}$-acetylglucosamine (GlcNAc) from the UDP-GlcNAc precursor to the hydroxyl group of serine and threonine residues, resulting in O-linked oligosaccharides in $T$. cruzi mucins [55]. Comparative genomic hybridization among T. cruzi strains also shows gene CNV in another gene involved in the synthesis of glycans on T. cruzi mucins, the beta-galactofuranosyl transferase genes $[8,56]$. Mucins are heavy glycosylated glycoproteins, and their glycan content may account for up to $60 \%$ of the total mucin weight $[55,57]$. These proteins are the most abundant component on the T. cruzi surface, covering the whole parasite with approximately $2 \times 10^{6}$ copies per cell $[39,55]$. Mucins are responsible for protecting the parasite from both the vector and the mammal defensive mechanisms and ensure the anchorage point and invasion of specific cells and tissues [55]. One of the forces driving the expansion of chromosome 31 in all the $T$. cruzi strains may be the need to glycosylate this large number of proteins that cover the parasite surface and are directly involved in parasite survival in both invertebrate and vertebrate hosts. Although chromosome 31 was also expanded in several Leishmania species [34], we found no large syntenic regions between $T$. cruzi and Leishmania chromosome 31, suggesting that the chromosome 31 expansion in Leishmania is driven by different evolutionary pressures.

\section{Conclusions}

It is well known that T. cruzi, as well as Leishmania and $T$. brucei, relies on gene duplication to increase the expression levels of key genes and to allow the generation of novel genes without loss of function [8, 20, 28, 36, 38-40]. Our study highlights the genome-wide CCNV in T. cruzi as a new level of gene expansion mechanism, allowing the rapid generation of diversity within the parasite. The estimation of chromosomal aneuploidies based on the RDC of single-copy genes comes as a new approach to evaluate the CCNV in T. cruzi, reducing the bias of repetitive and gap regions in the analysis and improving chromosomal comparisons between DTUs. As previously observed in Leishmania [34], aneuploidy appears to be well tolerated in trypanosomatids, due to their predominantly asexual replication mechanism. The chromosome copy number can vary considerably between strains from different $T$. cruzi DTUs and even within the same DTU. TcI appears to be more stable, and TcII had large differences between its strains, suggesting that this mechanism is widely used by the parasite to expand groups of genes. One of the limitations of our approach is that we are not able to investigate the karyotype structure of each strain, and the analysis is limited to comparing their differences based on the CL Brener predicted chromosomes because there are no other $T$. cruzi chromosomal sequences already 
published in the literature. Due to the extensive repetitive content, third-generation long read single-molecule sequencing is required not only to close the gaps in CL Brener chromosomes, but also to generate reliable chromosomal sequences of the other five DTUs allowing better CCNV estimations. Only three DTUs (TcI, II and III) were evaluated in this work. Therefore, it would be interesting to evaluate $\mathrm{CCNV}$ in the other T. cruzi DTUs, to better investigate this variation in the parasite. Finally, the expansion of chromosome 31, which is enriched with genes related to glycosylation pathways in all six strains evaluated, highlights the importance of this biochemical process to the parasite's survival.

\section{Methods}

\section{Parasite cloning in a semi-solid medium}

For cloning the T. cruzi Arequipa (TcI) and 231 (TcIII) strains, $10^{3}$ epimastigotes were plated into a semi-solid medium (low-melting agarose $0.75 \%$, brain heart infusion $48.4 \%$, liver infusion tryptose (LIT) $48.4 \%, 2.5 \%$ defibrinated blood, and $250 \mu \mathrm{g} / \mathrm{mL}$ penicillin/streptomycin) and incubated at $28{ }^{\circ} \mathrm{C}$ for 35 days. Single clones were obtained and transferred to $25-\mathrm{cm}^{3}$ culture flasks with $5 \mathrm{~mL}$ of LIT medium and $10 \%$ fetal bovine serum.

\section{Parasite culture and DNA isolation}

T. cruzi epimastigotes from Arequipa (TcI), Colombiana (TcI) and Y (TcII) strains were cultured in LIT medium supplemented with $10 \%$ fetal bovine serum. A total of $1 \times 10^{8}$ parasites from each strain were centrifuged at $3000 \mathrm{~g}$ in an Eppendorf 5804 Centrifuge. The parasites where washed three times with ice-cold PBS, suspended in PBS with $100 \mu \mathrm{g} / \mathrm{mL}$ proteinase $\mathrm{K}$ and incubated at $25{ }^{\circ} \mathrm{C}$ for $10 \mathrm{~min}$. The genomic DNA was obtained with the Wizard ${ }^{\circ}$ Genomic DNA Purification Kit (Promega) by following the manufacturer instructions. The DNA integrity was evaluated by agarose gel electrophoresis. The DNA samples were submitted to a genotyping protocol according to Souto et al., 1996 [58], de-Freitas et al., 2006 [15] and Burgos et al., 2007 [59].

\section{Genome sequencing}

A whole-genome shotgun library (WGSG) and sequencing of the T. cruzi Arequipa (TcI), Colombiana (TcI) and Y (TcII) strains were performed at the Computational Genomics Unity Darcy Fontoura de Almeida (UGCDFA) of the National Laboratory of Scientific Computation (LNCC) (Petrópolis, RJ, Brazil). For the 454 GS-FLX Titanium sequencing, each unpaired library was constructed using $5 \mu \mathrm{g}$ of genomic DNA (gDNA) and by following the GS FLX Titanium series protocols. All titrations, emulsions, PCR, and sequencing steps were carried out according to the manufacturer's protocol. One full PicoTiterPlate (PTP) was used for sequencing each library. In addition to the 454 sequencing, the Ion Proton ${ }^{\text {th }}$ was also used for unpaired sequencing. A total of $1 \mu \mathrm{g}$ of gDNA was used to prepare the deep sequencing libraries. All steps were also performed according to manufacturer's protocol. Reads from Sylvio (TcI) were kindly provided by Dr. Bjorn Andersson (Karolinska Institut). The 231 (TcIII) sequences were obtained using the Illumina Hiseq 2000 NGS platform (Baptista et al., in preparation). The Illumina and 454 read libraries from the Esmeraldo strain were downloaded from the National Center for Biotechnology Information (NCBI) (Additional file 6: Table S4).

\section{Preprocessing of reads}

The reads were checked for quality using the FASTQC tool (http://www.bioinformatics.babraham.ac.uk/projects/ fastqc/). Reads smaller than $30 \mathrm{nt}$ and with a Phred score lower than $20[60,61]$ were removed from the libraries using the fast_quality_trimmer, from the fastx toolkit ("http://hannonlab.cshl.edu/fastx_toolkit/index.html).

\section{Mapping and competitive mapping}

Whole genome shotgun reads from the T. cruzi Arequipa, Colombiana, Sylvio, Esmeraldo, Y, and 231 strains were mapped to the 41 chromosomes from both Esmeraldolike and non-Esmeraldo-like CL Brener haplotypes, version 6, downloaded from Tritrypdb (http://tritrypdb.org/ tritrypdb/). Mapping for each read library was performed simultaneously for both CL Brener haplotypes for competitive mapping, or separately for single mapping, using Bowtie 2 [62]. To account for the divergence between strains, the Bowtie2 preset "very sensitive" parameter was used, with the mismatch parameter changed to 1 . The competitive mapping was used to select the CL Brener haplotype closely related to each read library, and single mapping was used to estimate the CCNV in each strain. Mapped reads of each strain were filtered using a mapping quality threshold of 30 using SAMtools v1.1 [63]. The read depth coverage (RDC) for each position of each chromosome of the Esmeraldo-like and non-Esmeraldolike haplotypes for each $T$. cruzi strain was obtained by an in-house PERL script and BEDtools genomecov v2.16.2 [64]. The competitive mapping graphs were generated using GraphPad Prism V5.01 and scripts developed in PERL and R.

\section{Single-copy genes and chromosomal ploidy}

Ortholog genes between Esmeraldo-like and nonEsmeraldo-like CL Brener haplotypes were identified using OrthoMCL v2.0 [65], based on a combined approach of "reciprocal best hits" and a "Markov clustering algorithm" (MCL). Initially, an "all vs. all" local alignment using the BLASTp package 2.2.21 with an E-value of $1 \mathrm{e}-5$ as a cut-off was performed. The E-values were 
converted into $\log$ base to create the similarity matrix. An MCL with a 1.5 inflation parameter was applied to produce the ortholog clusters. A total of 1563 1:1 orthologs were selected and assumed to be single-copy genes in the haploid CL Brener genome (Additional file 1: Table S1). These single copy genes were selected to be used as chromosomal markers for unique genomic sequences, allowing CCNV estimations without the bias of repetitive regions. The mean RDC of the single-copy genes in each of the $41 \mathrm{CL}$ Brener chromosomes, based on the mapped reads from the Arequipa, Colombiana, Sylvio and 231 strains to the non-Esmeraldo-like haplotype, and the Esmeraldo and $\mathrm{Y}$ to the Esmeraldo-like haplotype, were generated by PERL scripts. The mean RDC of all single-copy genes in all chromosomes of each strain was assumed to be the genome coverage. The predicted copy number of each chromosome was determined based on the mean RDC of the single-copy genes in a given chromosome and normalized by the genome coverage. The genome coverage was estimated as $47 x$ for Arequipa, $28 \times$ for Colombiana, $9 \times$ for Sylvio, $52 \times$ for Esmeraldo, 34× for $\mathrm{Y}$ and $76 \times$ for 231 . The CCNV graphs were generated with GraphPad Prism V5.01 software and scripts in PERL and R.

\section{SNP content}

Single-nucleotide polymorphisms (SNPs) of the mapped reads from the single-copy genes in the T. cruzi Arequipa, Colombiana, Sylvio, Esmeraldo, Y and 231 strains to the CL Brener Esmeraldo-like and non-Esmeraldo-like haplotypes were obtained using the SAMtools function mpileup [63]. To reduce the chance of incorrectly identifying a SNP due to sequencing artifacts, we set the minimum number of mapped reads to 10 . To reduce the bias of collapsed regions, the maximum number of reads mapped in a SNP position was set to double the genome coverage of the corresponding genome. The SNP density for each strain was calculated and plotted using GraphPad Prism V5.01.

\section{Heterozygous SNPS}

Heterozygous SNPs between the CL Brener chromosome and the mapped reads for the six T. cruzi stains were obtained from the filtered SAMtools mpileup results [63]. To be considered as a reliable SNP, the position RDC must be at least 10. To reduce the bias of collapsed regions, the position RDC must also be lower than twice the genome coverage. For each chromosome, the proportion of the alleles in each predicted heterozygous site was obtained and rounded to the first decimal place. Base frequencies were rounded in ten categories, ranging from 0.1 to 1 , and an approximate distribution of base frequencies for each chromosome was plotted in R. To estimate the overall ploidy of each genome, the same methodology was applied, but the heterozygous positions from all CDSs from all chromosomes were employed simultaneously.

\section{Cluster dendrogram}

A hierarchical clustering analysis of all predicted T. cruzi chromosomal ploidy was performed using the Pvclust package [66] implemented in $\mathrm{R}$ (www.r-project.org) (R Development 2010). First, a distance matrix was built with pairwise Euclidean distances between the strains and the dendrogram was generated by the complete linkage method. To assess the uncertainty in hierarchical cluster analysis, we used the two bootstrap resampling methods implemented in Pvclust: bootstrap probability (BP) by ordinary bootstrap resampling and the approximately unbiased (AU) probability from multiscale bootstrap resampling, which provides better estimations than BP. Both methods were calculated with 10,000 iterations.

\section{Gene ontology}

Gene ontology categories that were significantly overrepresented in the genes of the CL Brener chromosome 31 were detected using the hypergeometric distribution analysis in BiNGO [67] with Benjamini and Hochberg false discovery rate correction.

\section{Availability of supporting data}

The Sequence Read Archives (SRA) supporting the results of this article are available in the NCBI GenBank repository, accession numbers: Arequipa(SRS838181), Colombiana(SRS841912), Esmeraldo(SRR833799, SRR83 3800, SRR058517, SRR058509, SRR058520, SRR058518, SRR058519, SRR058515, SRR058516, SRR058513, SRR05 8514, SRR058510, SRR058511, SRR058512) and Y(SRS8 42149). The 231 strain SRA is available ate the European Nucleotide Archive repository, by the accession number: PRJEB9129.

\section{Additional files}

Additional file 1: Table S1. T. cruzi CL Brener single copy genes,

Additional file 2: Table S2. Normalized RDC of the single copy genes from all six $T$. cruzi strains evaluated.

Additional file 3: Figure S1. Heterozygous SNPs proportion predicted ploidy of all the chromosomes from the T. cruzi strains: Arequipa, Colombiana, Sylvio, Esmeraldo, $Y$ and 231

Additional file 4: Figure S2. Normalized RDC of each position on the $41 \mathrm{CL}$ Brener chromosomes by the reads from the T. cruzi strains: Arequipa, Colombiana, Sylvio, Esmeraldo, Y and 231.

Additional file 5: Table S3. Gene ontology analysis of the Esmeraldolike and Non-Esmeraldo chromosome 31.

Additional file 6: Table S4. Trypanosoma cruzi strains sequencing information. 


\section{Authors' contributions}

$J L R C$ conceived the design of the study, performed all analyses, and draft the manuscript; GFRL, HRV, performed gene ontology analysis and participated in the chromosome copy number estimation analysis; TAOM performed the clustering analysis; RPB, GLM, RG, AMM, CB, RHG, CTL, BA, ATV participated in its design and helped to draft the manuscript; DCB conceived the study, its design and coordination and wrote the manuscript. All authors read and approved the final manuscript.

\section{Acknowledgements}

This study was funded by Fundação de Amparo a Pesquisa do Estado de Minas Gerais (FAPEMIG), Instituto Nacional de Ciência e Tecnologia de Vacinas (INCTV)-Conselho Nacional de Desenvolvimento Científico e Tecnológico (CNPq). DCB, AMM, ATV are CNPq research fellows. JLRC, GFRL, HRV and TAOM received scholarships from CAPES and RPB received a scholarship from CNPq. We thank Michele Silva de Matos, Alexandra Lehmkuhl Gerber and Laila Viana de Almeida for the technical support.

\section{Author details}

${ }^{1}$ Laboratório de Imunologia e Genômica de Parasitos, Departamento deParasitologia, Universidade Federal de Minas Gerais, Belo Horizonte, Brazil. ${ }^{2}$ Laboratório Nacional de Computação Científica, Petrópolis, Rio de Janeiro, Brazil. ${ }^{3}$ Departamento de Bioquímica e Imunologia, Universidade Federal de Minas Gerais, Belo Horizonte, Brazil. ${ }^{4}$ University of California San Francisco, San Francisco, CA, USA. ${ }^{5}$ Universidad Cayetano Heredia, Lima, MD, Peru. ${ }^{6}$ Johns Hopkins University, Baltimore, MD, USA. ${ }^{7}$ Department of Cell and Molecular Biology, Science for Life Laboratory, Karolinska Institutet, Stockholm, Sweden.

Received: 3 March 2015 Accepted: 1 June 2015 Published online: 04 July 2015

\section{References}

1. Hotez PJ, Bottazzi ME, Franco-Paredes C, Ault SK, Periago MR. The neglected tropical diseases of Latin America and the Caribbean: a review of disease burden and distribution and a roadmap for control and elimination. PLoS Negl Trop Dis. 2008;2(9):e300.

2. Coura JR, Dias JC. Epidemiology, control and surveillance of Chagas disease: 100 years after its discovery. Mem Inst Oswaldo Cruz. 2009;104 Suppl 1:31-40.

3. Martins-Melo FR, Alencar CH, Ramos Jr AN, Heukelbach J. Epidemiology of mortality related to Chagas' disease in Brazil, 1999-2007. PLoS Negl Trop Dis. 2012;6(2):e1508.

4. WHO. Research Priorities for Chagas Disease, Human African Trypanosomiasis and Leishmaniasis. Technical Report of the TDR Disease Reference Group on Chagas Disease, Human African Trypanosomiasis and Leishmaniasis. (Technical report series; no. 975). 2012. Availabe at: http://apps.who.int/iris/bitstream/10665/77472/1/WHO_TRS_975_eng.pdf.

5. Vargas N, Pedroso A, Zingales B. Chromosomal polymorphism, gene synteny and genome size in T. cruzi I and T. cruzi II groups. Mol Biochem Parasitol. 2004;138(1):131-41.

6. Lewis MD, Llewellyn MS, Gaunt MW, Yeo M, Carrasco HJ, Miles MA. Flow cytometric analysis and microsatellite genotyping reveal extensive DNA content variation in Trypanosoma cruzi populations and expose contrasts between natural and experimental hybrids. Int J Parasitol. 2009;39(12):1305-17.

7. Zingales B, Andrade SG, Briones MR, Campbell DA, Chiari E, Fernandes O, Guhl F, Lages-Silva E, Macedo AM, Machado CR, et al A new consensus for Trypanosoma cruzi intraspecific nomenclature: second revision meeting recommends Tcl to TcVI. Mem Inst Oswaldo Cruz. 2009;104(7):1051-4.

8. Minning TA, Weatherly DB, Flibotte S, Tarleton RL. Widespread, focal copy number variations (CNV) and whole chromosome aneuploidies in Trypanosoma cruzi strains revealed by array comparative genomic hybridization. BMC Genomics. 2011;12:139.

9. Ackermann AA, Panunzi LG, Cosentino RO, Sanchez DO, Aguero F. A genomic scale map of genetic diversity in Trypanosoma cruzi. BMC Genomics. 2012;13:736

10. Zingales B, Miles MA, Campbell DA, Tibayrenc M, Macedo AM, Teixeira MM, Schijman 627 AG, Llewellyn MS, Lages-Silva E, Machado CR, et al The revised Trypanosoma cruzi subspecific nomenclature: rationale, epidemiological relevance and research applications. Infection, genetics and evolution. 2012;12(2):240-53.
11. Panunzi LG, Aguero F. A genome-wide analysis of genetic diversity in Trypanosoma cruzi intergenic regions. PLoS Negl Trop Dis. 2014;8(5):e2839.

12. Tibayrenc M, Kjellberg F, Ayala FJ. A clonal theory of parasitic protozoa: the population structures of Entamoeba, Giardia, Leishmania, Naegleria, Plasmodium, Trichomonas, and Trypanosoma and their medical and taxonomical consequences. Proc Natl Acad Sci U S A. 1990;87(7):2414-8.

13. Tibayrenc M, Ayala FJ. How clonal are Trypanosoma and Leishmania? Trends Parasitol. 2013;29(6):264-9.

14. Westenberger SJ, Barnabe C, Campbell DA, Sturm NR. Two hybridization events define the population structure of Trypanosoma cruzi. Genetics. 2005;171(2):527-43.

15. de Freitas JM, Augusto-Pinto L, Pimenta JR, Bastos-Rodrigues L, Goncalves VF, Teixeira SM, Chiari E, Junqueira AC, Fernandes O, Macedo AM, et al. Ancestral genomes, sex, and the population structure of Trypanosoma cruzi. PLoS Pathog. 2006;2(3):e24

16. Machado CA, Ayala FJ. Nucleotide sequences provide evidence of genetic exchange among distantly related lineages of Trypanosoma cruzi. Proc Nat Acad Sci U S A. 2001;98(13):7396-401.

17. Brisse S, Henriksson J, Barnabe C, Douzery EJ, Berkvens D, Serrano M, De Carvalho MR, Buck GA, Dujardin JC, Tibayrenc M. Evidence for genetic exchange and hybridization in Trypanosoma cruzi based on nucleotide sequences and molecular karyotype. Infection, genetics and evolution. 2003;2(3):173-83.

18. Sturm NR, Vargas NS, Westenberger SJ, Zingales B, Campbell DA. Evidence for multiple hybrid groups in Trypanosoma cruzi. Int J Parasitol. 2003;33(3):269-79.

19. Baptista RD, D'Avila DA, Segatto M, Do Valle IF, Franco GR, Valadares HMS Gontijo ED, Galvao LMD, Pena SDJ, Chiari E, et al Evidence of substantial recombination among Trypanosoma cruzi II strains from Minas Gerais. Infect Genet Evol. 2014;22:183-91.

20. El-Sayed NM, Myler PJ, Bartholomeu DC, Nilsson D, Aggarwal G, Tran AN Ghedin E, Worthey EA, Delcher AL, Blandin G, et al The genome sequence of Trypanosoma cruzi, etiologic agent of Chagas disease. Science. 2005;309(5733):409-15

21. Franzen O, Ochaya S, Sherwood E, Lewis MD, Llewellyn MS, Miles MA, Andersson B. Shotgun sequencing analysis of Trypanosoma cruzi I Sylvio X10/1 and comparison with T. cruzi VI CL Brener. PLoS Negl Trop Dis. 2011;5(3):e984.

22. Souza RT, Lima FM, Barros RM, Cortez DR, Santos MF, Cordero EM, Ruiz JC, Goldenberg S, Teixeira MM, da Silveira JF. Genome size, karyotype polymorphism and chromosomal evolution in Trypanosoma cruzi. PLoS One. 2011;6(8):e23042

23. Cerqueira GC, Bartholomeu DC, DaRocha WD, Hou L, Freitas-Silva DM, Machado CR, El-Sayed NM, Teixeira SM. Sequence diversity and evolution of multigene families in Trypanosoma cruzi. Mol Biochem Parasitol. 2008;157(1):65-72.

24. Stranger BE, Forrest MS, Dunning M, Ingle CE, Beazley C, Thorne N, Redon R, Bird $C P$, de Grassi $A$, Lee $C$, et al Relative impact of nucleotide and copy number variation on gene expression phenotypes. Science. 2007;315(5813):848-53.

25. Iskow RC, Gokcumen O, Lee C. Exploring the role of copy number variants in human adaptation. Trends in genet. 2012;28(6):245-57.

26. Martins C, Baptista CS, lenne S, Cerqueira GC, Bartholomeu DC, Zingales B. Genomic organization and transcription analysis of the 195-bp satellite DNA in Trypanosoma cruzi. Mol Biochem Parasitol. 2008;160(1):60-4.

27. Clayton CE. Life without transcriptional control? From fly to man and back again. EMBO J. 2002;21(8):1881-8.

28. Martinez-Calvillo S, Vizuet-de-Rueda JC, Florencio-Martinez LE, Manning-Cela RG, Figueroa-Angulo EE. Gene expression in trypanosomatid parasites. J Biomed Biotechnol. 2010;2010:525241.

29. Henriksson J, Dujardin JC, Barnabe C, Brisse S, Timperman G, Venegas J, Pettersson U, Tibayrenc M, Solari A. Chromosomal size variation in Trypanosoma cruzi is mainly progressive and is evolutionarily informative. Parasitology. 2002;124(Pt 3):277-86.

30. Pedroso A, Cupolillo E, Zingales B. Evaluation of Trypanosoma cruzi hybrid stocks based on chromosomal size variation. Mol Biochem Parasitol. 2003;129(1):79-90.

31. Triana O, Ortiz S, Dujardin JC, Solari A. Trypanosoma cruzi: variability of stocks from Colombia determined by molecular karyotype and minicircle Southern blot analysis. Exp Parasitol. 2006;113(1):62-6.

32. Lima FM, Souza RT, Santori FR, Santos MF, Cortez DR, Barros RM, Cano MI, Valadares HM, Macedo AM, Mortara RA, et al Interclonal variations in the molecular karyotype of Trypanosoma cruzi: chromosome rearrangements in a single cell-derived clone of the G strain. PLoS One. 2013;8(5):e63738. 
33. Weatherly DB, Boehlke C, Tarleton RL. Chromosome level assembly of the hybrid Trypanosoma cruzi genome. BMC Genomics. 2009;10:255.

34. Rogers MB, Hilley JD, Dickens NJ, Wilkes J, Bates PA, Depledge DP, Harris D Her $Y$, Herzyk P, Imamura $H$, et al Chromosome and gene copy number variation allow major structural change between species and strains of Leishmania. Genome Res. 2011;21(12):2129-42.

35. Branche C, Ochaya S, Aslund L, Andersson B. Comparative karyotyping as a tool for genome structure analysis of Trypanosoma cruzi. Mol Biochem Parasitol. 2006;147(1):30-8.

36. El-Sayed NM, Myler PJ, Blandin G, Berriman M, Crabtree J, Aggarwal G, Caler E, Renauld H, Worthey EA, Hertz-Fowler C, et al Comparative genomics of trypanosomatid parasitic protozoa. Science. 2005;309(5733):404-9.

37. Tibayrenc M, Ward P, Moya A, Ayala FJ. Natural populations of Trypanosoma cruzi, the agent of Chagas disease, have a complex multiclonal structure. Proc Natl Acad Sci U S A. 1986;83(1):115-9.

38. Bartholomeu DC, Cerqueira GC, Leao AC, daRocha WD, Pais FS, Macedo C, Djikeng A, Teixeira SM, El-Sayed NM. Genomic organization and expression profile of the mucin-associated surface protein (masp) family of the human pathogen Trypanosoma cruzi. Nucleic Acids Res. 2009;37(10):3407-17.

39. De Pablos LM, Osuna A. Multigene Families in Trypanosoma cruzi and Their Role in Infectivity. Infect Immun. 2012;80(7):2258-64.

40. Bartholomeu DC, de Paiva RM, Mendes TA, DaRocha WD, Teixeira SM. Unveiling the intracellular survival gene kit of trypanosomatid parasites. PLoS Pathog. 2014;10(12):e1004399.

41. Sheltzer JM, Blank HM, Pfau SJ, Tange Y, George BM, Humpton TJ, Brito IL, Hiraoka Y, Niwa O, Amon A. Aneuploidy drives genomic instability in yeast. Science. 2011;333(6045):1026-30.

42. Abbey D, Hickman M, Gresham D, Berman J. High-Resolution SNP/CGH Microarrays Reveal the Accumulation of Loss of Heterozygosity in Commonly Used Candida albicans Strains. G3 (Bethesda) 2011;1(7):523-30.

43. Rancati G, Pavelka N, Fleharty B, Noll A, Trimble R, Walton K, Perera A, Staehling-Hampton K, Seidel CW, Li R. Aneuploidy underlies rapid adaptive evolution of yeast cells deprived of a conserved cytokinesis motor. Cell. 2008;135(5):879-93.

44. Farrer RA, Henk DA, Garner TW, Balloux F, Woodhams DC, Fisher MC Chromosomal copy number variation, selection and uneven rates of recombination reveal cryptic genome diversity linked to pathogenicity. PLoS Genet. 2013;9(8):1003703.

45. Peacock L, Ferris V, Sharma R, Sunter J, Bailey M, Carrington M, Gibson W. Identification of the meiotic life cycle stage of Trypanosoma brucei in the tsetse fly. Proc Natl Acad Sci U S A. 2011;108(9):3671-6.

46. Akopyants NS, Kimblin N, Secundino N, Patrick R, Peters N, Lawyer P, Dobson DE, Beverley SM, Sacks DL. Demonstration of genetic exchange during cyclical development of Leishmania in the sand fly vector. Science. 2009;324(5924):265-8.

47. Gaunt MW, Yeo M, Frame IA, Stothard JR, Carrasco HJ, Taylor MC, Mena SS, Veazey P, Miles GA, Acosta N, et al Mechanism of genetic exchange in American trypanosomes. Nature. 2003;421(6926):936-9.

48. Sturm NR, Campbell DA. Alternative lifestyles: the population structure of Trypanosoma cruzi. Acta Trop. 2010;115(1-2):35-43.

49. Llewellyn MS, Miles MA, Carrasco HJ, Lewis MD, Yeo M, Vargas J, Torrico F, Diosque $P$, Valente $V$, Valente SA, et al Genome-scale multilocus microsatellite typing of Trypanosoma cruzi discrete typing unit I reveals phylogeographic structure and specific genotypes linked to human infection. PLoS Pathog. 2009;5(5):e1000410

50. Augusto-Pinto L, Teixeira SMR, Pena SDJ, Machado CR. Single-nucleotide Polymorphisms of the Trypanosoma cruzi MSH2 gene support the existence of three phylogenetic lineages presenting differences in mismatch-repair efficiency. Genetics. 2003;164(1):117-26.

51. Machado CR, Augusto-Pinto L, McCulloch R, Teixeira SM. DNA metabolism and genetic diversity in Trypanosomes. Mutat Res. 2006;612(1):40-57.

52. Kanmogne GD, Bailey M, Gibson WC. Wide variation in DNA content among isolates of Trypanosoma brucei ssp. Acta Trop. 1997;63(2-3):75-87.

53. Ghedin E, Bringaud F, Peterson J, Myler P, Berriman M, Ivens A, Andersson B, Bontempi E, Eisen J, Angiuoli S, et al Gene synteny and evolution of genome architecture in trypanosomatids. Mol Biochem Parasitol. 2004;134(2):183-91.

54. de Lederkremer RM, Agusti R. Glycobiology of Trypanosoma cruzi. Adv Carbohydr Chem Biochem. 2009;62:311-66.
55. Buscaglia CA, Campo VA, Frasch ACC, Di Noia JM. Trypanosoma cruzi surface mucins: host-dependent coat diversity. Nat Rev Microbiol. 2006:4(3):229-36.

56. Jones C, Todeschini AR, Agrellos OA, Previato JO, Mendonca-Previato L. Heterogeneity in the biosynthesis of mucin O-glycans from Trypanosoma cruzi tulahuen strain with the expression of novel galactofuranosylcontaining oligosaccharides. Biochemistry. 2004;43(37):11889-97.

57. Acosta-Serrano A, Almeida IC, Freitas-Junior LH, Yoshida N, Schenkman S. The mucin-like glycoprotein super-family of Trypanosoma cruzi: structure and biological roles. Mol Biochem Parasitol. 2001;114(2):143-50.

58. Souto RP, Fernandes O, Macedo AM, Campbell DA, Zingales B. DNA markers define two major phylogenetic lineages of Trypanosoma cruzi. Mol Biochem Parasitol. 1996;83(2):141-52.

59. Burgos JM, Altcheh J, Bisio M, Duffy T, Valadares HM, Seidenstein ME, Piccinali R, Freitas JM, Levin MJ, Macchi L, et al. Direct molecular profiling of minicircle signatures and lineages of Trypanosoma cruzi bloodstream populations causing congenital Chagas disease. Int J Parasitol. 2007;37(12):1319-27.

60. Ewing B, Hillier L, Wendl MC, Green P. Base-calling of automated sequencer traces using phred. I. Accuracy assessment genome res. 1998;8(3):175-85.

61. Richterich P. Estimation of errors in "raw" DNA sequences: a validation study. Genome Res. 1998;8(3):251-9.

62. Langmead B, Salzberg SL. Fast gapped-read alignment with Bowtie 2. Nat Methods. 2012;9(4):357-9.

63. Li H, Handsaker B, Wysoker A, Fennell T, Ruan J, Homer N, Marth G, Abecasis G, Durbin R. The Sequence Alignment/Map format and SAMtools. Bioinformatics. 2009;25(16):2078-9.

64. Quinlan AR, Hall IM. BEDTools: a flexible suite of utilities for comparing genomic features. Bioinformatics. 2010;26(6):841-2.

65. Li L, Stoeckert Jr CJ, Roos DS. OrthoMCL: identification of ortholog groups for eukaryotic genomes. Genome Res. 2003;13(9):2178-89.

66. Suzuki R, Shimodaira H. Pvclust: an R package for assessing the uncertainty in hierarchical clustering. Bioinformatics. 2006;22(12):1540-2.

67. Maere S, Heymans K, Kuiper M. BiNGO: a Cytoscape plugin to assess overrepresentation of gene ontology categories in biological networks. Bioinformatics. 2005;21(16):3448-9.

\section{Submit your next manuscript to BioMed Central and take full advantage of:}

- Convenient online submission

- Thorough peer review

- No space constraints or color figure charges

- Immediate publication on acceptance

- Inclusion in PubMed, CAS, Scopus and Google Scholar

- Research which is freely available for redistribution

Submit your manuscript at www.biomedcentral.com/submit
C Biomed Central 